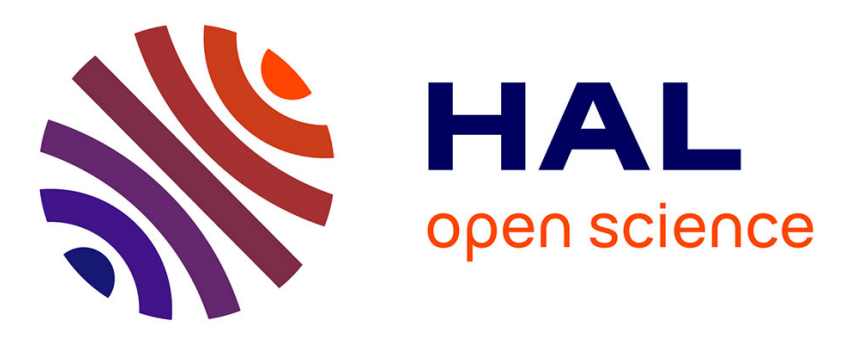

\title{
Studies on the cytochrome b 6 / f complex. I. Characterization of the complex subunits in Chlamydomonas reinhardtii
}

Claire Lemaire, Jacqueline Girard-Bascou, Francis-André Wollman, Pierre Bennoun

\section{To cite this version:}

Claire Lemaire, Jacqueline Girard-Bascou, Francis-André Wollman, Pierre Bennoun. Studies on the cytochrome b $6 / \mathrm{f}$ complex. I. Characterization of the complex subunits in Chlamydomonas reinhardtii. Biochimica biophysica acta (BBA) - Bioenergetics, 1986, 851 (2), pp.229-238. 10.1016/0005-2728(86)90130-1 . cea-02946507

\section{HAL Id: cea-02946507 https://hal-cea.archives-ouvertes.fr/cea-02946507}

Submitted on 23 Sep 2020

HAL is a multi-disciplinary open access archive for the deposit and dissemination of scientific research documents, whether they are published or not. The documents may come from teaching and research institutions in France or abroad, or from public or private research centers.
L'archive ouverte pluridisciplinaire HAL, est destinée au dépôt et à la diffusion de documents scientifiques de niveau recherche, publiés ou non, émanant des établissements d'enseignement et de recherche français ou étrangers, des laboratoires publics ou privés. 


\title{
Studies on the cytochrome $b_{6} / f$ complex. I. Characterization of the complex subunits in Chlamydomonas reinhardtii
}

\author{
Claire Lemaire, Jacqueline Girard-Bascou, Francis-André Wollman \\ and Pierre Bennoun
}

\author{
Service de Photosynthèse, Institut de Biologie Physico-Chimique, 13 rue Pierre et Marie Curie, \\ 75231 Paris Cedex 05 (France)
}

(Received February 10th, 1986)

Key words: Cytochrome $b_{6} / f$ complex; Peroxidase; Thylakoid membrane; Protein assembly; Chloroplast biogenesis

We have analyzed the heme-associated peroxidase activity in thylakoid membranes from the, green algae Chlamydomonas reinhardtii after electrophoresis in the presence of sodium dodecyl sulfate. Besides cytochrome $f$ and cytochrome $b_{6}$, we observed peroxidase activity in two other bands, of 34 and $11 \mathrm{kDa}$, of unknown origin. Characterization of the $b_{6} / f$ complex subunits was undertaken by means of a comparison of the polypeptide deficiencies in several $b_{6} / f$ mutants with the polypeptide content of preparations enriched in $b_{6} / f$ complexes. We conclude that the $b_{6} / f$ complex consists of five subunits. Using site-specific translation inhibitors, we show that cytochrome $f$, cytochrome $b_{6}$ and subunit IV are of chloroplast origin, whereas the Rieske protein and probably subunit $V$ are translated on cytoplasmic ribosomes. A model of assembly of the complex is proposed: a cytochrome moiety, comprising the subunits of chloroplast origin, is assembled in the thylakoid membranes prior to the insertion and assembly of the subunits encoded in the nuclear genome.

\section{Introduction}

In recent years, there have been several reports on the purification of $b_{6} / f$ complexes from higher plants [1-4] and cyanobacteria [5]. Cytochromes $f$ and $b_{6}$, and the Rieske protein have been identified among the putative subunits of the isolated complexes. The cytochromes were characterized by their redox differential absorption spectra and by heme staining upon SDS-polyacrylamide gel electrophoresis of the complex, whereas the Fe-S center-containing Rieske protein was identified by its characteristic EPR signal at $g=1.89$. The use

\footnotetext{
Abbreviations: Cyt, cytochrome; PS, Photosystem; HP, high potential; LP, low potential; $\alpha$, antibody; TMBZ, 3,3',5,5'-tetramethylbenzidine; DCMU, 3-(3,4-dichlorophenyl)-1,1-dimethylurea; PBS, phosphate-buffered saline; $F_{0}$, initial yield of fluorescence; $F_{\max }$, maximal yield of fluorescence.
}

of antibodies against these subunits have proved useful for their identification in thylakoid membranes from different species and in subsequent preparations enriched in $b_{6} / f$ complexes [6-8].

There is a controversy about the number of additional subunits in the $b_{6} / f$ complex. Besides a polypeptide in the 14-18 $\mathrm{kDa}$ region, quoted as subunit IV and supposedly related to cytochrome $b_{6}[9,10]$, polypeptides in the $35 \mathrm{kDa}$ region and polypeptides of molecular weights smaller than 10 $\mathrm{kDa}$ were observed in some instances $[2,3,5]$.

Analysis of the pattern of polypeptide deficiencies in mutants lacking the $b_{6} / f$ complex can be regarded as an alternative approach to the identification of the polypeptides constitutive of the complex. The absence of the main subunits of the $b_{6} / f$ complex was reported in mutants from Maize [11] and Lemna [12]. Maroc and Garnier [13] described mutants from Chlamydomonas rein- 
hardtii lacking the two cytochromes, but they did not report on the possible absence of other polypeptides.

In the present study, we attempted to characterize the subunits of the $b_{6} / f$ complex from $C$. reinhardtii through a comparison of the polypeptide content of preparations, enriched in $b_{6} / f$ complexes, with polypeptide deficiencies in several photosynthesis mutants altered in this complex. We have successfully used a similar approach for the identification of the subunits of protein complexes containing the PS I and PS II reaction centers $[14,15]$. The sites of synthesis of the various subunits were investigated by pulse-labelling experiments in the presence of site-specific translation inhibitors. The comparative analysis of the pulse-labeled thylakoids from the mutant strains and the wild type gave some insight on the mode of assembly of the complex in the thylakoid membrane.

\section{Materials and Methods}

Mutant strains. In this study, we analysed several mutants from $C$. reinhardtii, showing altered $b_{6} / f$ complexes, with respect to the wild type and, in some instances, to the FuD112 mutant lacking in PS II reaction centers [16]. The mutant strains, blocked in the electron transfer chain at the level of the $b_{6} f$ complex, are listed below.

- The nuclear mutant, ac21, and the chloroplast mutant, FuD2, were isolated and characterized by Levine and Smillie [17] and Bennoun et al. [18], respectively. Contrarily to the other mutants described in this paper, the FuD2 mutant still grows in phototrophic conditions although at reduced rates as compared to the wild type.

The chloroplast mutants, FuD4, FuD6, FuD8, and the nuclear mutant, F18, were obtained after mutagenesis with 5-fluorodeoxyuridine for the former [18], and 5-fluorouracyl for the latter [14].

The enrichment step was performed according to Schmidt et al. [19] as modified by Bennoun and Delepelaire [20]. The $b_{6} / f$ mutants were screened by their high stationary level of fluorescence as described by Bennoun and Levine [21]. They display fluorescence characteristics similar to that of the FuD2 mutant previously described by Bennoun et al. [18]: same $F_{0}$ as in the wild type and a continuous fluorescence rise with no decay phase.
Their $F_{\text {max }}$ level is identical to that observed in the presence of DCMU in wild-type cells.

The genetic analysis of the mutants was performed according to Levine and Ebersold [22]. We analysed an average of 12 tetrads per cross and checked the nuclear segregation of the mating type. For chloroplast mutations, the transmission was almost exclusively from the mating type + parental strain, while a $2: 2$ segregation was observed for all nuclear mutations.

Methods. All the strains were grown at $25^{\circ} \mathrm{C}$ in Tris-acetate-phosphate medium at a light intensity of $300 \mathrm{~lx}$. The cells were harvested in late exponential phase $\left(4 \cdot 10^{6}\right.$ cells $\left./ \mathrm{ml}\right)$, except for pulse-labelling experiments $\left(2 \cdot 10^{6}\right.$ cells $\left./ \mathrm{ml}\right)$.

Pulse labelling was carried out according to Delepelaire [23]. Purified thylakoid membranes were prepared according to Chua and Bennoun [24]. Preparations enriched in $b_{6} / f$ complex were obtained by a procedure similar to that of Pick and Racker [25], using thylakoid membranes from the wild type or FuD50 mutant lacking the chloroplast ATPase [26], solubilized in the absence of $\left(\mathrm{NH}_{4}\right)_{2} \mathrm{SO}_{4}$ by a mixture of $1 \%$ octylglucoside and $0.5 \%$ cholate. After fractionation of the supernatant by $\left(\mathrm{NH}_{4}\right)_{2} \mathrm{SO}_{4}$ precipitation, and sucrose gradient centrifugation, the upper reddish brown band was collected and analysed for heme content after SDS-polyacrylamide gel electrophoresis.

Electrophoresis were run in the Laemmli system [27] using 7.5-15\% acrylamide gradients (referred to as a 'conventional gel system' in the present paper) or $12-18 \%$ acrylamide gradients in the presence of $8 \mathrm{M}$ urea [28]. Heme staining was performed according to Thomas et al. [29]. Polypeptides were stained by Coomassie blue or detected by autoradiography of the dried gels using Agfa-Gevaert industrial P films. Molecular weights of the polypeptides were estimated using a low molecular-weight calibration kit from Pharmacia.

Electrophoretic transfers were carried out according to Towbin et al. [30]. Protein saturation of the nitrocellulose sheets was performed using either $0.2 \%$ tween and $0.25 \%$ gelatin in phosphatebuffered saline or $5 \%$ low-fat milk in phosphatebuffered saline. Binding of the $\alpha-$ Rieske and $\alpha-b_{6}$, generously provided by G. Hauska, was detected using radioiodinated protein $A$ according to Burnette [31]. 


\section{Results}

In order to characterize the heme deficiencies in the $b_{6} / f$ mutants we first compared the heme content of the thylakoid membranes from $C$. reinhardtii and spinach by TMBZ staining of urea gels (Fig. 1A). Both types of thylakoid displayed the same two bands of apparent molecular weight, 41000 and 23000 , in this gel system. In conventional SDS-polyacrylamide gel electrophoresis they had an apparent molecular weight of 38000 and 25000 (not shown). On the other hand we noted the presence of two stained bands in $C$. reinhardtii, quoted $h_{1}$ and $h_{2}$ on Fig. 1A, of apparent molecular weights, $34 \mathrm{kDa}$ and $11 \mathrm{kDa}$, respec-

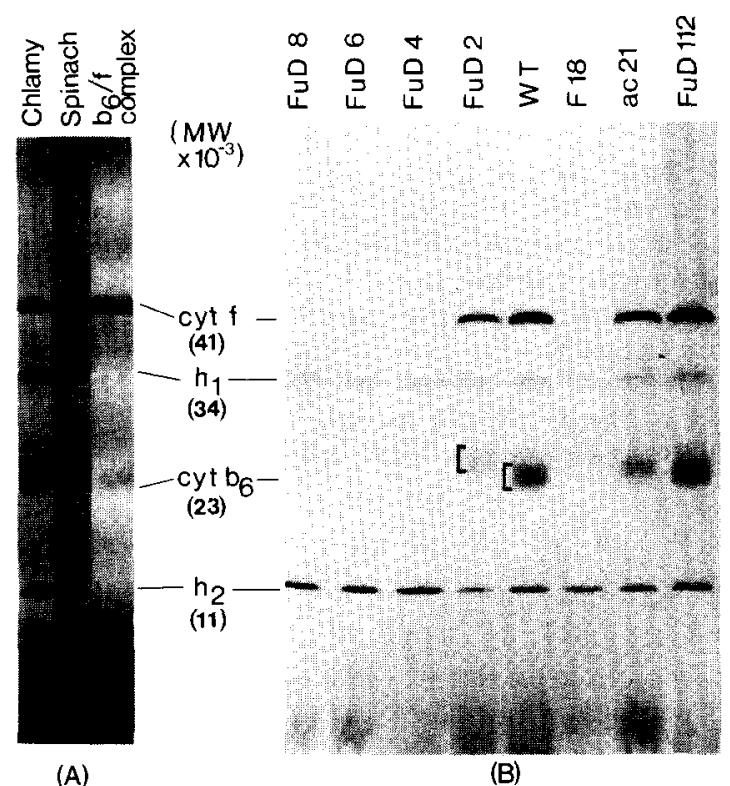

(A)

(B)

Fig. 1. Heme staining of polypeptides from purified thylakoids and from a $b_{6} / f$ complex preparation, using urea SDS polyacrylamide gel electrophoresis (A) comparison of a $b_{6} / f$ complex preparation with thylakoid membranes from the wild type of $C$. reinhardtii and from spinach. Cyt $f$ and $C y t b_{6}$ are observed in the three samples. Note the presence of two heme stainable bands. $h_{1}$ and $h_{2}$, in thylakoid membranes from $C$. reinhardtii. (B) comparison between thylakoids from different strains of $C$. reinhardtii. Cyt $f$ and $C y t b_{6}$ are still visible in the ac21 mutant, but are absent in the FuD4, FuD8 and F18 mutants. Traces of Cyt $f$ are detected in the FuD6 mutant. The FuD2 mutant contains Cyt $f$ and a modified Cyt $b_{6}$. Note the presence of $h_{1}$ and $h_{2}$ in all strains, including the FuD112 mutant lacking PS II reaction centers. WT, wild type. tively. In spinach thylakoids, $h_{2}$ was never observed whereas a faint staining in the position of $h_{1}$ could be detected in some instances. The stained bands of 38 and $25 \mathrm{kDa}$ (41 and $23 \mathrm{kdA}$, respectively, in urea gels) have been previously attributed in spinach to Cyt $f$ and Cyt $b_{6}$, respectively [1]. Accordingly, purified $b_{6} / f$ complexes from $C$. reinhardtii contained these two bands (Fig. 1A), whereas most of the $b_{6} / f$ mutants showed no heme staining in these electrophoretic positions (Fig. 1B). In contrast, $h_{1}$ and $h_{2}$ were absent in the $b_{6} / f$ complex preparations but they remained in the various $b_{6} / f$ mutants that we have characterized. These bands were still visible in mutants lacking in PS II reaction centers (FuD112 on Fig. 1B). Therefore they do not correspond to Cyt $b-559_{\mathrm{HP}}$ which is structurally associated with PS II reaction centers [32] and is lacking in PS II mutants (Wollman, F.-A., unpublished results).

Three types of $b_{6} / f$ mutant could be distinguished on the basis of their heme content:

- mutants devoid of Cyt $f$ and Cyt $b_{6}$, like FuD4, FuD8 and F18, or showing only traces of Cyt $f$, like FuD6;

- mutants displaying a heme-staining pattern similar to that in the wild type, like ac21;

- mutants showing a modification in the electrophoretic position of one of the two cytochromes. This was the case of the FuD2 mutant which contains a somewhat modified Cyt $b_{6}$.

We first analysed the group of mutants devoid of both cytochromes. After Coomassie blue staining of urea gels (Fig. 2), we noted the absence of the $41 \mathrm{kDa}$ polypeptide, corresponding to Cyt $f$, in the four mutants thought to lack the $b_{6} / f$ complex on the basis of their heme-staining pattern. This group of mutants showed two other deficiencies in the 23 and $19.5 \mathrm{kDa}$ positions (see inset at the right-hand corner of Fig. 2). The former deficiency corresponds to the position where Cyt $b_{6}$ was detected by the heme-staining procedure used in Fig. 1. No other modifications were observed among the population of polypeptides of still lower molecular weight, which is best resolved with this gel system.

We then performed immunoblotting experiments in order to check the absence of the Rieske protein in these mutants. This is shown on Figs. 3 and 4, for the F18 mutant taken as typical of the 


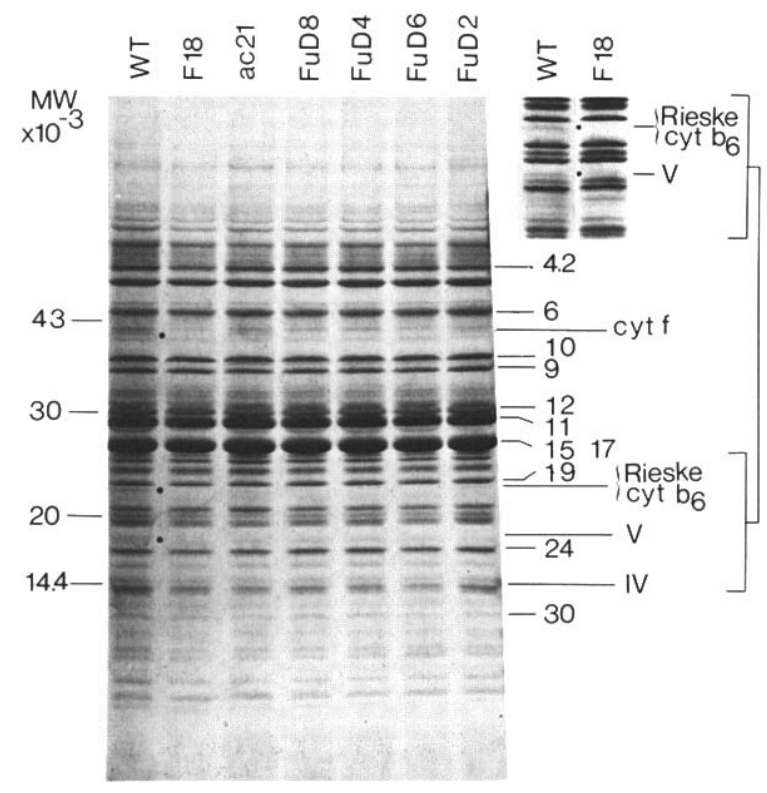

Fig. 2. Polypeptide patterns of thylakoid membranes from wild type (WT) and $b_{6} / f$ mutant strains from $C$. reinhardtii, using urea-SDS polyacrylamide gel electrophoresis after Coomassie blue staining. Polypeptides are numbered according to Bennoun et al. [15]. Besides the absence of Cyt $f$ in several mutants, two polypeptide deficiencies are observed in the 23 and $19 \mathrm{kDa}$ regions. Comparison of these regions in the F18 and wild type thylakoid membranes is shown the insert at the right upper corner.

first group of mutants in the above classification. In spinach as well as in the wild-type of $C$. reinhardtii the $\alpha$-Rieske labeled a single band of 23 $\mathrm{kDa}$ in the urea gel system. No labeling was observed in the F18 mutant (Fig. 3A). When similar experiments were performed with a $\alpha$-Cyt $b_{6}$, a $23 \mathrm{kDa}$ band was also labeled in spinach and in the wild type of $C$. reinhardtii. This label was absent in the F18 mutant (Fig. 3B). That these observations revealed a comigration of the Rieske protein and Cyt $b_{6}$ in this gel system, and not a cross-reaction of the antibodies, is illustrated on Fig. 4: in conventional gel systems (i.e., in the absence of urea) the two proteins migrated at different positions giving rise to a label in the 25 $\mathrm{kDa}$ region with the $\alpha-\mathrm{Cyt} b_{6}$ and in the $19 \mathrm{kDa}$ region with the $\alpha$-Rieske. The absence of radiolabeled bands in these positions in the F18 thylakoids confirmed the absence of the two antigens in this type of mutants.

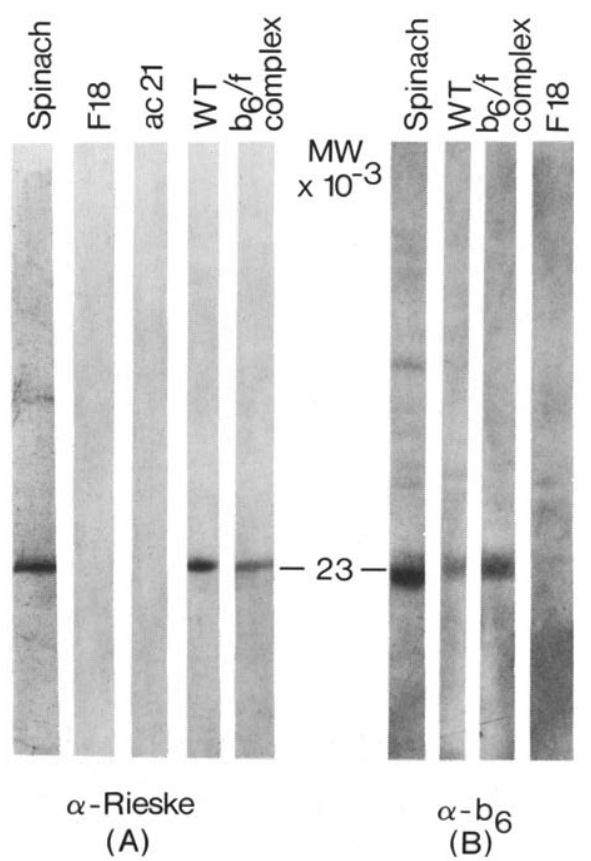

Fig. 3. Immunoblots on a $b_{6} / f$ complex preparation and on thylakoid membranes from wild type (WT) and $b_{6} / f$ mutant strains after urea-SDS polyacrylamide gel electrophoresis. Using $\alpha$-Rieske protein (A) and $\alpha$-Cyt $b_{6}$ (B) coupled to radioiodinated protein $A$. Note that $23 \mathrm{kDa}$ bands are labeled with the two antibodies in spinach and $C$. reinhardtii wild-type thylakoids as well as in the $b_{6} / f$ complex preparation. No such labeling is observed in the F18 mutant. The ac21 mutant shows no $23 \mathrm{kDa}$ labeling with the $\alpha$-Rieske only.

In agreement with their heme staining pattern, the ac21 and FuD2 mutants contained Cyt $f$, corresponding to the $41 \mathrm{kDa}$ polypeptide in the urea gel shown on Fig. 2. However, the $23 \mathrm{kDa}$ band was lacking in the ac21 mutant and was present in the FuD2 mutant. Immunoblotting experiments (Figs. 3 and 4) showed that the Rieske protein was lacking in the ac21 mutant and was present in the FuD2 mutant, whereas $\mathrm{Cyt} b_{6}$ was present in both strains with a modified electrophoretic position in the case of the FuD2 mutant. These observations indicated that the $23 \mathrm{kDa}$ band, revealed by Coomassie blue staining of urea gels, corresponded to the Rieske protein only. Therefore, it should be emphasized that Cyt $b_{6}$ is not stained efficiently enough to be detected among the polypeptides of the thylakoid membrane.

As was the case with the mutants lacking the 


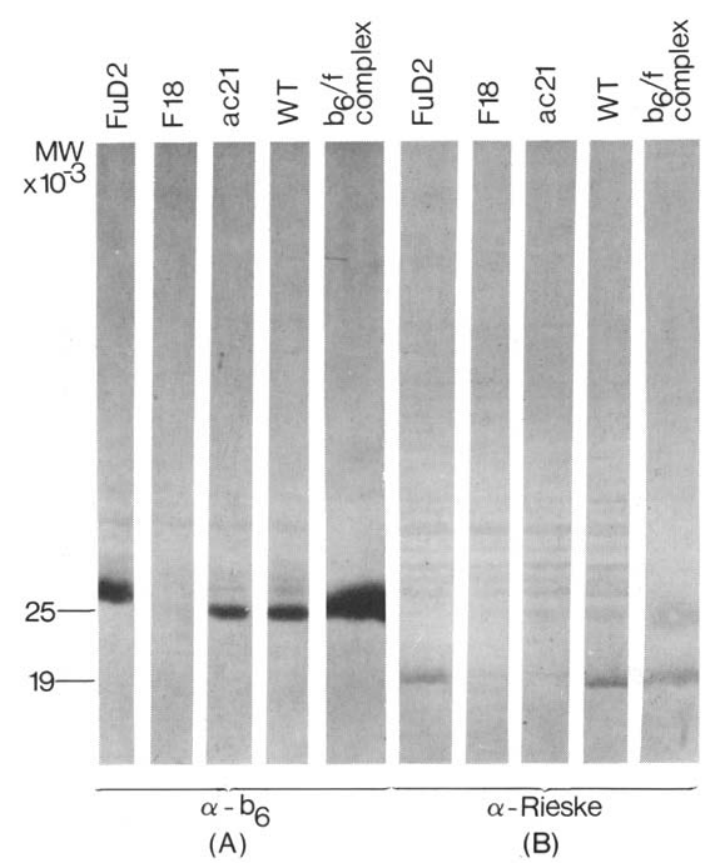

Fig. 4. Same as Fig. 3 after SDS polyacrylamide gel electrophoresis. Distinct bands are labeled by the $\alpha$-Rieske protein (19 kDa) and the $\alpha$-cytb6 (25 kDa). Note the absence of labeling with $\alpha$-Rieske protein in the ac21 mutant and the absence labeling by the $\alpha$-Rieske protein and $\alpha$-Cyt $b_{6}$ in the F18 mutant. WT, wild type.

whole $b_{6} / f$ complex, thylakoids from the ac21 mutant were lacking in a $19.5 \mathrm{kDa}$ polypeptide in the urea gel system (Fig. 2). This polypeptide was still present in the FuD2 thylakoids, although showing a modified electrophoretic mobility in some experiments.

In order to understand if the $b_{6} / f$ complex consisted of only the four polypeptides deficient in the $b_{6} / f$ mutants, we attempted to purify the complex from $C$. reinhardtii. Preparations enriched in $b_{6} / f$ complexes showed a $b_{6} / f$ ratio of 1.76 (using millimolar extinction coefficients, $\epsilon_{543-553}$, of 21 for Cyt $f$ [33] and $\epsilon_{563-570}$ of 14 for Cyt $b_{6}$ [34]). Similar experiments performed on intacts thylakoids yielded a $b_{6} / f$ ratio of 1.94 . Therefore, our $b_{6} / f$ complex preparations showed a slight deficiency $(\approx 10 \%)$ in Cyt $b_{6}$. The polypeptide content of this preparation is shown on Fig. 5. In conventional SDS-polyacrylamide gel electrophoresis (Fig. 5A), Cyt $f$ appeared as a main band

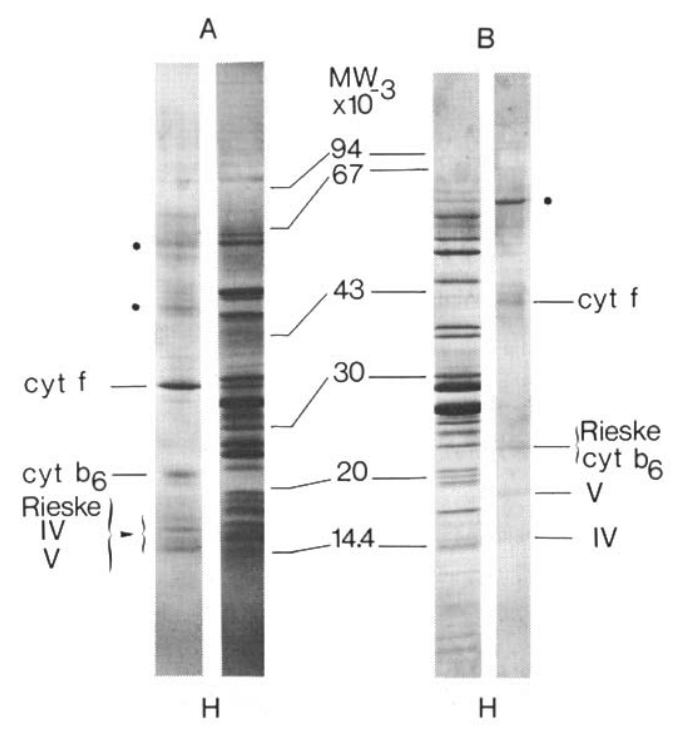

Fig. 5. Comparison of the polypeptide patterns in preparations enriched in $b_{6} / f$ complexes and in thylakoid membranes from the wild-type strain. (A) Heated samples after SDS polyacrylamide gel electrophoresis. (B) Heated samples after ureaSDS-polyacrylamide gel electrophoresis. The asterisk indicates contaminant polypeptides of high molecular weight, the amount of which varied from one preparation to another.

in the $38 \mathrm{kDa}$ region. Among the other polypeptides present in this preparation were a $25 \mathrm{kDa}$ polypeptide, comigrating with the heme staining due to Cyt $b_{6}$ and recognized by $\alpha$-Cyt $b_{6}$ (Fig. 4A), a $19 \mathrm{kDa}$ polypeptide and a doublet of about $16 \mathrm{kDa}$. The $19 \mathrm{kDa}$ band was labeled by the $\alpha$-Rieske (Fig. 4B). A rather different pattern was observed in urea gels (Fig. 5B): besides the 41 $\mathrm{kDa}$ band, attributed by heme staining to Cyt $f$ (Fig. 1), we observed only three bands, of apparent molecular weights of 23000,19500 and 16000. As discussed above, Cyt $b_{6}$ and the Rieske protein migrated in the position of the $23 \mathrm{kDa}$ band (see Figs. 1 and 3). Other polypeptides of high molecular weight, thought to be contaminants of the $b_{6} / f$ complex, since their amounts varied from one preparation to another, are clearly visible in the two gel systems of Fig. 5A and B.

Thus the isolated $b_{6} / f$ complex contained the four polypeptides lacking in the $b_{6} / f$ mutants. However, a fifth polypeptide, of $16 \mathrm{kDa}$ in urea gels, was also visible in these preparations (see Fig. 5B). It could be either a contaminant or a 
constitutive subunit of the complex. In the latter case, the absence of modifications in the $16 \mathrm{kDa}$ region of the polypeptide patterns of the mutants should be attributed to a comigration of several polypeptides, masking the absence of the $b_{6} / f$ subunit.

In an attempt to distinguish between comigrating polypeptides in this region, on the basis of possible differences in their origin of synthesis, we performed pulse labeling experiments in the presence of site-specific translation inhibitors on whole cells of the wild type and $b_{6} / f$ mutants of $C$. reinhardtii. These experiments were also designed to determine the site of synthesis of the four subunits deficient in most of the mutants, and could be used in the identification of subunit IV, known as a chloroplast-encoded subunit from the work of Alt et al. [7]. To this end, we have isolated thylakoid membranes from cells pulse-labeled for $45 \mathrm{~min}$ with ${ }^{14} \mathrm{C}$-acetate in the presence of cycloheximide (Fig. 6), which inhibits cytoplasmic translation, or chloramphenicol (Fig. 7.), which inhibits chloroplast translation. It is worth mentioning that these experiments give access to the insertion of individual subunits of the complex in the thylakoid membranes of the mutants, even though they would not assemble or accumulate to the same extent as in the wild-type membranes $[35,16]$.

Three subunits of the $b_{6} / f$ complex - namely Cyt $f$, Cyt $b_{6}$ and subunit IV - have been reported as chloroplast translates in higher plants [7]. The chloroplast translates in the thylakoids of C. reinhardtii are shown on Fig. 6. The use of conventional gels (left of Fig. 6) allowed us to observe the insertion of Cyt $b_{6}$, during the time of the pulse, in all the strains we have analysed. Only did the FuD2 mutant show insertion of an abnormal Cyt $b_{6}$, with an apparent molecular weight about $1 \mathrm{kDa}$ larger than usual. Cyt $f$ was lacking from the thylakoid membranes of the FuD4 mutant, but it was inserted in the thylakoids from the other strains in which the complex does not accumulate (see for instance the F18 or FuD6 mutants in Fig. 6). The urea gel system on the right of Fig. 6 shows the absence of insertion of a $16 \mathrm{kDa}$ polypeptide in the FuD6 thylakoids. Insertion of this $16 \mathrm{kDa}$ polypeptide occurred in the other mutant strains (not shown). Thus, the 16

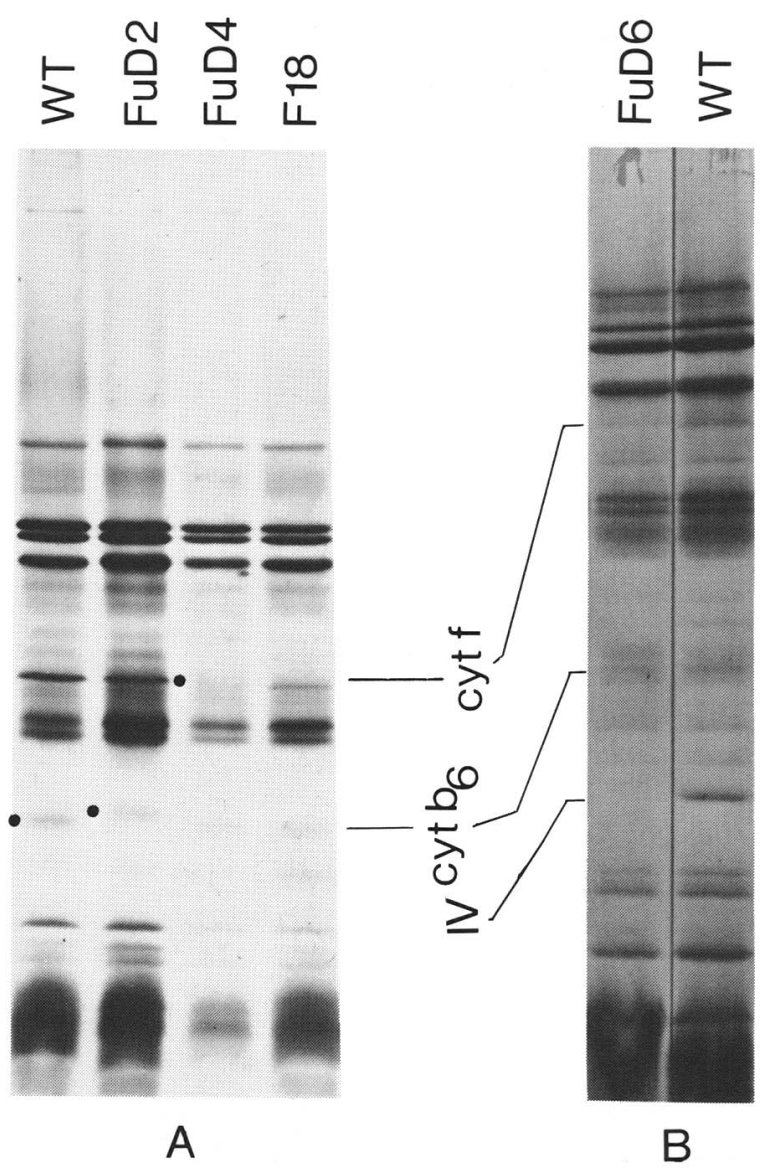

Fig. 6. Autoradiograms of chloroplast translates, inserted in the thylakoid membranes from wild-type (WT) and $b_{6} / f$ mutant strains, viewed after SDS polyacrylamide gel electrophoresis (A) and urea-SDS polyacrylamide gel electrophoresis (B). Cells were pulse-labeled for $45 \mathrm{~min}$ with $\left[{ }^{14} \mathrm{C}\right]$ acetate, in the presence of cycloheximide. Note the modified position of Cyt $b_{6}$ in the FuD2 mutant and the absence of Cyt $f$ and of subunit IV in the FuD4 and FuD6 mutants, respectively.

$\mathrm{kDa}$ polypeptide that we observed in the polypeptide pattern of the $b_{6} / f$ complex (Fig. 5) is probably of chloroplast origin. Therefore, it should correspond to subunit IV in the $b_{6} / f$ complex of C. reinhardtii.

The polypeptides, translated on cytoplasmic ribosomes and inserted in the thylakoid membranes during the time of our pulse labeling, are shown on the urea gel of Fig. 7. There was no detectable insertion of the $23 \mathrm{kDa}$ polypeptide, corresponding to the Rieske protein, in the ac21 nuclear mutant which contains Cyt $f$ and Cyt $b_{6}$. On the contrary the $23 \mathrm{kDa}$ band was visible in 


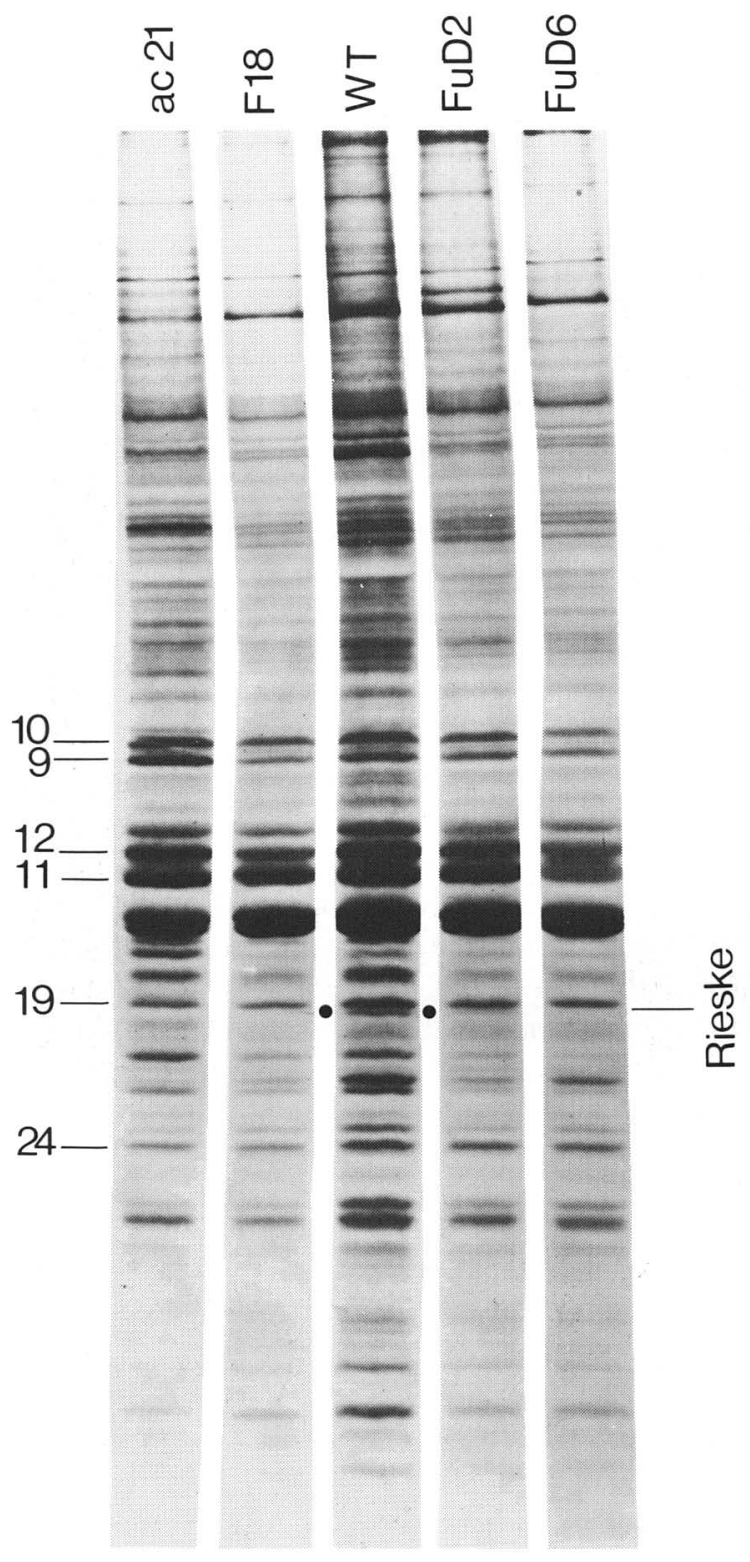

Fig. 7. Autoradiograms of cytoplasmic translates, inserted in the thylakoid membranes from the wild-type (WT) and $b_{6} / f$ mutant strains, viewed after urea-SDS polyacrylamide gel electrophoresis. Note the absence of the Rieske protein in all mutants but FuD2.

the pulse-labeled thylakoids from the FuD2 mutant, as expected from the immunoblotting experiments and from its polypeptide pattern after Coomassie blue staining. More striking is the observation that the Rieske protein was not inserted in the thylakoid membranes from any of the other mutants (see for instance F18 and FuD6 in Fig. 7).

Although a $19.5 \mathrm{kDa}$ polypeptide was clearly missing in the mutant thylakoids after Coomassie blue staining (Fig. 2), we observed no deficiencies in labeled polypeptides in this region in urea gels whether the pulse labeling experiments were performed in the presence of chloramphenicol or in the presence of cycloheximide (see Figs. 6 and 7). It was therefore impossible to determine, in these experiments, the site of synthesis of the fifth subunit of the $b_{6} / f$ complex from $C$. reinhardtii. However, indirect evidence for its nuclear origin arises from the absence of labeled polypeptides in the $19.5 \mathrm{kDa}$ region in the thylakoids from the wild type when pulse labeled with cycloheximide (Fig. 6).

\section{Discussion}

Polypeptides showing peroxidase activity in the thylakoid membrane of . reinhardtii

As previously reported by Maroc and Garnier [13], Cyt $f$ and Cyt $b_{6}$ from $C$. reinhardtii were easily identified among thylakoid polypeptides after TMBZ staining of polyacrylamide gels. We showed that thylakoid membranes of $C$. reinhardtii contained two other stained bands, quoted $\mathrm{h}_{1}$ and $\mathrm{h}_{2}$ in this paper, distinct from Cyt $f$ and Cyt $b_{6}$, since they remained in the $b_{6} / f$ mutants. Although the procedure might detect peroxidase activities due to prosthetic groups other than the hemes, we looked for a possible correspondance between $\mathrm{h}_{1}$ and $\mathrm{h}_{2}$ and Cyt $b-559, \mathrm{Hp}$ or LP, and Cyt $b-560$, recently detected by Bendall and Sanguansermsri (personal communication). We can rule out the first possibility, since we observed $h_{1}$ and $h_{2}$ in thylakoid membranes from a mutant lacking in PS II reaction centers although PS II mutants are devoid of Cyt $b-559_{\mathrm{HP}}$. These PS II mutants would also lack Cyt $b-560$, but would still contain significant amounts of Cyt $b-559_{\mathrm{LP}}$ (Bendall and Sanguansermsri, personal communication). Therefore, among the cytochromes already identified in the thylakoid membranes from C. reinhardtii, Cyt $b-559_{\mathrm{LP}}$ is the more likely to correspond to either $h_{1}$ and $h_{2}$. Such correlations clearly require further investigations. Still another 
possibility is the participation of $h_{1}$ and $h_{2}$ in the chlororespiratory pathway described by Bennoun [36] in $C$. reinhardtii. The activity of the putative oxidase, responsible for the reoxidation of the plastoquinone pool in this pathway, was reported to be independent from the presence or absence of $b_{6} / f$ complexes [37]. $h_{1}$ and $h_{2}$ might then be related to the oxidase itself or to a cytochrome complex, specific of the chlororespiratory pathway.

Characterization of the subunits of the $b_{6} / f$ complex

We have identified five subunits in the $b_{6} / f$ complex from the green algae $C$. reinhardtii. The complex comprised Cyt $f$ and Cyt $b_{6}$, which are chloroplast translates, the Rieske protein, translated on cytoplasmic ribosomes, and two smaller subunits. That Cyt $b_{6} / f$ complexes from $C$. reinhardtii contain three non-heme subunits in addition to Cyt $f$ and Cyt $b_{6}$ has also been observed by Bendall and Sanguansermsri (personal communication). The electrophoretic properties of the two cytochromes and of the Rieske protein showed minor differences in Spinach and $C$. reinhardtii. These observations arose from the comparison of the positions of the heme-stained bands and from immunoblotting experiments performed on the thylakoids from the two organisms. The apparent molecular weights of these subunits varied from one gel system to another. Therefore we consider that the differences in their molecular weights as reported by different groups (reviewed in Ref. 38) may not be significant. In conventional SDS-polyacrylamide gel electrophoresis, Cyt $f$ was found in the 33-38 $\mathrm{kDa}$ region, Cyt $b_{6}$ was in the 19-25 $\mathrm{kDa}$ region and the Rieske protein was about 19-20 kDa. However, we observed marked changes in this electrophoretic pattern when 12-18\% acrylamide gradients in the presence of $8 \mathrm{M}$ urea were used. Cyt $f$ migrated with a higher apparent molecular weight $(41 \mathrm{kDa})$, whereas Cyt $b_{6}$ and the Rieske protein comigrated with an apparent molecular weight of $23 \mathrm{kDa}$. This is worth mentioning, since two other subunits of the $b_{6} / f$ complex, with apparent molecular weights, 19.5 and 16 $\mathrm{kDa}$, were resolved in this type of gel and could be misquoted as the Rieske protein and subunit IV.

It has been previously reported that subunit IV from spinach has an apparent molecular of about $17 \mathrm{kDa}$ (2) and is encoded by the chloroplast genome [7]. Out of the two small subunits that we identified in the $b_{6} / f$ complex from $C$. reinhardtii, only the $16 \mathrm{kDa}$, resolved in urea gels, was shown unambiguously to be translated on chloroplast ribosomes. Therefore, its correspondence with subunit IV from spinach is very likely.

The role of the $19.5 \mathrm{kDa}$ subunit, quoted as subunit $\mathrm{V}$ in this paper, remains to be elucidated. Owing to the absence of a labeled band, in this region of molecular weights, in the pattern of thylakoid polypeptides translated on chloroplast ribosomes, we believe that subunit $\mathrm{V}$ is translated

TABLE I

POLYPEPTIDES OF CHLOROPLAST OR CYTOPLASMIC ORIGIN, ACCUMULATED (A) OR INSERTED (B) IN THE THYLAKOID MEMBRANES

+ , polypeptides present; -, polypeptides below detection; ND, not determined; Chlp, chloroplast; Cytp, cytoplasmic.

\begin{tabular}{|c|c|c|c|c|c|c|c|c|c|c|}
\hline & \multicolumn{5}{|l|}{ A } & \multicolumn{5}{|l|}{ B } \\
\hline & $\begin{array}{l}\text { Cyt } f \\
\text { (Chlp) }\end{array}$ & $\begin{array}{l}\text { Cyt } b_{6} \\
\text { (Chlp) }\end{array}$ & $\begin{array}{l}\text { IV } \\
\text { (Chlp) }\end{array}$ & $\begin{array}{l}\text { Rieske } \\
\text { (Cytp) }\end{array}$ & $\begin{array}{l}\text { V } \\
\text { (Cytp?) }\end{array}$ & $\begin{array}{l}\text { Cyt } f \\
\text { (Chlp) }\end{array}$ & $\begin{array}{l}\text { Cyt } b_{6} \\
\text { (Chlp) }\end{array}$ & $\begin{array}{l}\text { IV } \\
\text { (Chlp) }\end{array}$ & $\begin{array}{l}\text { Rieske } \\
\text { (Cytp) }\end{array}$ & $\begin{array}{l}\text { V } \\
\text { (Cytp?) }\end{array}$ \\
\hline Wild type & + & + & ND & + & + & + & + & + & + & ND \\
\hline FuD2 & + & $+a$ & ND & + & + & + & $+a$ & + & + & ND \\
\hline ac21 & + & + & ND & - & - & + & + & + & - & ND \\
\hline FuD4 & - & - & ND & - & - & - & + & + & - & ND \\
\hline FuD8 & - & - & ND & - & - & + & + & + & - & ND \\
\hline F18 & - & - & ND & - & - & + & + & + & - & ND \\
\hline FuD6 & $+/-b$ & - & ND & - & - & $+1-$ & + & - & - & ND \\
\hline
\end{tabular}

\footnotetext{
a Polypeptide present in a modified position.

b Polypeptide in a markedly reduced amount.
} 
in the cytoplasm. Preliminary experiments, using preparations enriched in $b_{6} / f$ complexes after pulse labelling the cells of $C$. reinhardtii with inhibitors of chloroplast translation, favor this hypothesis. It should be noted that plastocyanin is also of low molecular weight, $10 \mathrm{kDa}$ [39], and is translated in the cytoplasm [40]. However, thylakoids from the ac208 mutant from C. reinhardtii, lacking in plastocyanin [41], still showed a $19.5 \mathrm{kDa}$ polypeptide upon urea-SDS-polyacrylamide gel electrophoresis (unpublished observation). It is then unlikely that subunit $\mathrm{V}$ corresponded to some plastocyanin retained in the isolated complex. That such subunits of low molecular weight may exist in $b_{6} / f$ complexes from higher plants has been previously mentioned $[2,5]$.

\section{The assembly of the $b_{6} / f$ complex}

The comparison of the polypeptide content in the various mutants gave some insight on the mode of assembly of the $b_{6} / f$ complexes in the thylakoid membranes of $C$. reinhardtii. We can hypothesize at least three steps in the assembly of a multiple subunit membrane protein: the insertion of the subunits in the membrane, their accumulation and subsequent assembly in a functional membrane protein complex. Our data, relevant to these processes in the case of the $b_{6} / f$ complex, are gathered on Table I. The majority of the mutants that we analysed in the present paper, lack the five subunits of the complex. However, one mutant, the ac21, was deficient only in the Rieske protein and in subunit $\mathrm{V}$. These observations tend to define two parts in the complex: a cytochrome moiety, comprising Cyt $f, \mathrm{Cyt} b_{6}$ and subunit IV, which can accumulate and assemble in the membrane in the absence of the other part of the complex, consisting of the Rieske protein and subunit V. This latter part would not accumulate in the thylakoid membrane in the absence of the cytochrome moiety.

We observed that the subunits of the cytochrome moiety were inserted independently in thylakoids isolated from pulse-labeled cells of $C$. reinhardtii: for instance, Cyt $b_{6}$ and subunit IV were inserted in the absence of Cyt $f$ in the FuD4 mutant, whereas the two cytochromes were inserted in the absence of subunit IV in the FuD6 mutant. However the inserted subunits, in the two mutants, did not accumulate to the same extent as in the wild-type thylakoids. Only did Cyt $f$ show a slight accumulation in the absence of subunit IV in the FuD6 mutant. These observations support a concerted accumulation of the subunits by a mechanism probably involving the assembly of the newly inserted subunits in the thylakoid membranes. The other part of the complex, exemplified by the case of Rieske protein, could not be detected, by pulse labeling, in the membranes of the mutants showing no accumulation of the cytochrome moiety. This indicates that the insertion of the Rieske subunit was dependent on the preceding accumulation of the cytochrome moiety and further substantiates the existence of an assembly between to parts of the $b_{6} / f$ complex.

Thus we propose the following mode of assembly of $b_{6} / f$ complexes in the thylakoid membranes: as a first step, the subunits encoded in the chloroplast genome - Cyt $b_{6}$ and subunit IV which show sequence homologies with Cyt $b$ from mitochrondria [9], and Cyt $f-$ are inserted independently, then assembled in a cytochrome moiety which allows the subsequent insertion and concomitant assembly of the subunits encoded in the nuclear genome (the Rieske protein and subunit V). It is worth mentioning that a similar situation probably prevails in the assembly of the subunits of the PS II complex: the chloroplast encoded subunits were functionally assembled in a PS II mutant from $C$. reinhardtii devoid of most of the nuclear-encoded subunits which participate in the formation of the $\mathrm{O}_{2}$-evolving site [15]. The reverse situation was never observed and could be easily interpreted by the fact that the $\mathrm{O}_{2}$-evolving site subunits are peripheral membrane proteins [35]. In this respect, the case of the $b_{6} / f$ complex is somewhat different, since the Rieske protein has been described as a transmembrane protein $[42,43]$.

\section{Acknowledgements}

We thank D. Bendall for fruitful exchanges of experimental observations. P. and A. Joliot for stimulating discussions and G. Hauska for his encouragement at the beginning of this work and for the use of his antibodies. C. Lemaire is a recipient of te Agence Française pour la Maîtrise de l'Énergie. This work was supported by the 
CNRS (ATP génétique des algues, contract no. 2221).

\section{References}

1 Hurt, E. and Hauska, G. (1981) Eur. J. Biochem. 117, 591-599

2 Hurt, E. and Hauska, G. (1982) J. Bioenerg. Biomembranes $14,405-424$

3 Phillips, A.L. and Gray, J.C. (1983) Eur. J. Biochem. 137, 553

4 Clark, R.D. and Hind, G. (1983) J. Biol. Chem. 258, 10348-10354

5 Krinner, M., Hauska, G., Hurt, E. and Lockau, W. (1982) Biochim. Biophys. Acta 681, 110-117

6 Hurt, E., Hauska, G. and Malkin, R. (1981) FEBS Lett. $134,1-5$

7 Alt, J., Westhoff, P., Sears, B.B., Nelson, N., Hurt, E., Hauska, G. and Herrmann, G. (1983) EMBO J. 2, 979-986

8 Bullerjahn, G.S., Riethman, H.C. and Sherman, L.A. (1985) Biochim. Biophys. Acta 810, 148-157

9 Widger, W.R., Cramer, W.A. Herrmann, R.G. and Trebst, A. (1984) Proc. Natl. Acad. Sci. USA 81, 674-678

10 Heinemeyer, W., Alt. J. and Herrmann, R.G. (1984) Curr. Genetics 8 543-549

11 Metz, J.G., Milles, D. and Rutherford, A.W. (1983) Plant Physiol. 73, 452-459

12 Lam, E. and Malkin, R. (1985) Biochim. Biophys. Acta 810, 106-109

13 Maroc, J. and Garnier, J. (1981) Biochim. Biophys. Acta 637, 473-480

14 Girard, J., Chua, N.H. Bennoun, P., Schmidt, G. and Delosme, M. (1980) Curr. Genetics 2, 215-221

15 Bennoun, P., Diner, B., Wollman, F.-A. Schmidt, G.W. and Chua, N.H. (1981) in Photosynthesis, Vol. III (Akoyunoglou, G., ed.), pp. 839-849, Balaban International Science Services, Philadelphia, PA

16 Bennoun, P., Spierer-Herz, M., Erickson, J., Girard-Bascou, J., Pierre, Y. Delosme, M. and Rochaix, J.D. (1986) Plant Mol. biol., in the press

17 Levine, R.P. and Smillie, R.M. (1962) Proc. Natl. Acad. Sci. USA 48, 417-421

18 Bennoun, P., Masson, A., Piccioni, R. and Chua, N.H. (1978) in Chloroplast Development (Akoyunoglou, G. and Argyroudi-Akoyunoglou, J.H., eds.), pp. 721-726, Elsevier/North-Holland Biomedical Press, Amsterdam
19 Schmidt, G.W., Matlin, K.S. and Chua, N.H. (1977) Proc. Natl. Acad. Sci. USA 74, 610-614

20 Bennoun, P. and Delepelaire, P. (1982) in Chloroplast Molecular Biology (M., Hallick, R.B. and Chua, N.-H., eds.) pp. 25-38, Elsevier Biomedical Press, Amsterdam

21 Bennoun, P. and Levine, R.P. (1967) Plant Physiol. 42, 1284-1287

22 Levine, R.P. and Ebersold, W.T. (1960) Annu. Rev. Microbiol. 14, 197-214

23 Delepelaire, P. (1983) Photochem. and Photobiophys. 6, 279-291

24 Chua, N.-H. and Bennoun, P. (1975) Proc. Natl. Acad. Sci. USA 72, 2175-2179

25 Pick, U. and Racker, E. (1979) J. Biol. Chem. 254, 2793-2799

26 Woessner, J.P., Masson, A., Harris, E.H., Bennoun, P., Gilham, N.W. and Boynton, J.E. (1984) Plant Mol. Biol. 3, $177-190$

27 Laemmli, U.K. (1970) Nature (Lond.) 227, 680-685

28 Piccioni, R.G., Bennoun P. and Chua, N.H. (1981) Eur. J. Biochem. 117, 93-102

29 Thomas, P.E., Ryan, D. and Levin, W. (1976) Anal. Bioch. $75,168-176$

30 Towbin, H., Staehelin, T. and Gordon, J. (1979) Proc. Natl. Acad. Sci. USA 76, 4350-4354

31 Burnette, W.N. (1981) Anal. Biochem. 112, 195-203

32 Satoh, K. (1985) Photochem. and Photobiol. 42, 845-853

33 Nelson, N. and Neumann, J. (1972) J. Biol. Chem. 247, $1817-1824$

34 Stuart A.L. and Wasserman, A.R. (1973) Biochim. Biophys. Acta 314, 284-297

35 Delepelaire, P. (1984) EMBO J. 3, 701-706

36 Bennoun, P. (1982) Proc. Natl. Acad. Sci. USA 79, 4352-4356

37 Bennoun, P. (1983) FEBS Lett. 156, 363-365

38 Hauska, G., Hurt, E., Gabellini, N. and Lockau, W. (1983) Biochim. Biophys. Acta 726, 97-133

39 Bohner, H., Bohme, H. and Boger, P. (1981) Febs Lett. 131, 386-388

40 Haslett, B.G. and Cammack, R. (1974) Biochem. J. 144, $567-573$

41 Gorman, D.S. and Levine, R.P. (1965) Proc. Natl. Acad. Sci. USA 54, 1665-1669

42 Mansfield, R.W. and Anderson, J.M. (1985) Biochim. Biophys. Acta $809,435-444$

43 Ortiz, W. and Malkin, R. (1985) Biochim. Biophys. Acta $808,164-170$ 